\title{
Spatio-temporal Scanning and Statistical Test of the Accelerating Moment Release (AMR) Model Using Australian Earthquake Data
}

\author{
Yucang Wang ${ }^{1,2}$, Can Yin $^{1}$, Peter Mora ${ }^{1}$, Xiang-Chu Yin ${ }^{2,3}$, and Keyin Peng ${ }^{3}$
}

\begin{abstract}
The Accelerating Moment Release (AMR) preceding earthquakes with magnitude above 5 in Australia that occurred during the last 20 years was analyzed to test the Critical Point Hypothesis. Twelve earthquakes in the catalog were chosen based on a criterion for the number of nearby events. Results show that seven sequences with numerous events recorded leading up to the main earthquake exhibited accelerating moment release. Two occurred near in time and space to other earthquakes preceded by AMR. The remaining three sequences had very few events in the catalog so the lack of AMR detected in the analysis may be related to catalog incompleteness. Spatio-temporal scanning of AMR parameters shows that $80 \%$ of the areas in which AMR occurred experienced large events. In areas of similar background seismicity with no large events, 10 out of 12 cases exhibit no AMR, and two others are false alarms where AMR was observed but no large event followed. The relationship between AMR and LoadUnload Response Ratio (LURR) was studied. Both methods predict similar critical region sizes, however, the critical point time using AMR is slightly earlier than the time of the critical point LURR anomaly.
\end{abstract}

Key words: Critical Point Hypothesis, Accelerating Moment Release (AMR) model, earthquake prediction, Load-Unload Response Ratio (LURR), Australia earthquakes.

\section{Introduction}

In recent years, increased intermediate magnitude seismicity before large earthquakes has been widely noted (SYKES and JAUME, 1990; KEILIS-BOROK et al., 1988; KNOPOFF et al., 1996). Since these events often occur in an area that is considerably larger than what elasto-dynamic interactions would predict, some researchers suggested that large earthquakes are analogous to a critical point (Sornette and Sammis, 1995; Saleur et al., 1996). According to the Critical Point Hypothesis (CPH), the Earth's crust does not remain perpetually in or near a critical state, and a large event moves the system away from the critical state into a stable

${ }^{1}$ QUAKES, Department of Earth Sciences, The University of Queensland, Qld 4072, Brisbane, Australia. E-mail: wangyc@quakes.uq.edu.au; canyon@quakes.uq.edu.au; mora@quakes.uq.edu.au

${ }^{2}$ LNM, Institute of Mechanics, Chinese Academy of Sciences, Beijing, 100080, China.

E-mail: xcyin@public.bta.net.cn

${ }^{3}$ Center for Analysis and Prediction, China Seismological Bureau, Beijing, 100036, China.

E-mail: xcyin@public.bta.net.cn 
state with a relatively low seismicity. Subsequent accumulation of tectonic stress drives the system back towards the critical state. Accelerating seismic Moment Release (AMR) was cited as an indicator of a region approaching the critical state.

AMR has been tested by substantial researcher (BUFE and VARNES, 1993; BuFE et al., 1994; Bowman et al., 1998; Jaume and Sykes, 1999; Brehm and Braile, 1998, 1999; Robinson, 2000; PApazachos and PAPAZAChos, 2000), but mostly in inter-plate areas. One would ask: Can AMR be observed in different tectonic settings?. The purpose of this study is to test the Critical Point Hypothesis by studying AMR features statistically in a tectonic setting different from the settings previously observed. We use Australian seismicity data and retrospectively test its predictive capability. We mainly focus on the following questions: How many large earthquakes are preceded by AMR and how many would be missed by this method? How many regions exhibiting AMR experience a later large event? How many regions where AMR is seen are exhibiting false alarms, with no following large event? Since both AMR and LURR have been proposed as indicators that the crust within a region of study is approaching a critical state, we are also interested in the relation between AMR and Load-Unload Response Ratio (LURR) (YIN et al., 1995, 2000, 2002), for example, do AMR and LURR predict a similar time, magnitude and critical region size prior to a large earthquake?

\section{The Fitting Method and Australian Data}

Acceleration of seismic moment or Benioff strain, i.e., the square-root of seismic energy release, in moderate earthquakes prior to the large earthquakes can be modeled using a power-law time-to-failure relationship (VARNES, 1989; BUFE and VARNES, 1993, BUFE et al., 1994)

$$
\Omega(t)=A+B\left(t_{f}-t\right)^{m},
$$

where $\Omega$ is the cumulative Benioff strain at time $t, t_{f}$ is the time of the main event or critical time, $m$ is an exponent that defines the curvature of the power-law acceleration and $A$ and $B$ are empirical constants to be determined. Log-periodic corrections to equation (1) were introduced (SorNetTE and SAmmis, 1995; SALEUR et al., 1996). Since three additional parameters and extra complexity are introduced, and it requires data of high quality, only Eq. (1) is used.

A nonlinear least-square method is employed to fit Eq. (1) to the data in the present study. In order to determine the quality of the fit, a parameter $C$ (Bowman et al., 1998) is used

$$
C=\frac{\text { power law fit RMS error }}{\text { linear law fit RMS error }}
$$


The combination of parameters $A, B$ and $m$ in (1) that produce a minimum value of $C$ is regarded as the best fit. In searching for the best fit, we use a multi-parameter estimate method with the following steps:

(1) Fix a radius for the circular region and select earthquakes within this region, fix $t_{f}$ to the time of the main event, fix $m$ and initial time $t_{0}$.

(2) Fit Eq. (1) to the data to calculate $A$ and $B$, then determine the parameter $C$ according Eq. (2).

(3) Change the exponent $m$ from 0.25 to 1 , and change $t_{0}$ in a certain range, and repeat step (2) to find the combination of fit parameters which minimizes $C$.

(4) Change the radius and restart from step (1). The radius for which $m$ is minimal is regarded as the optimal (or critical) region size $R_{C}$.

Geoscience Australia seismic catalog is used in this study. The Australian continent lies wholly within the Indo-Australian Plate thus experiencing intra-plate seismicity. Figure 1a shows Australian M > 4.0 earthquakes since 1960.

According to JAUMÉ and SYKeS (1999), only events within two magnitude units of the main shock contribute towards AMR. This would suggest a cutoff of $M 3$ for the events with $5<M<5.5$ examined in this study. However, in most cases there were insufficient $M>3$ events prior to main shocks, consequently a lower cutoff of $M 2.5$ has been employed.

\section{Results}

\section{Statistical Test with Large Events}

AMR curves and optimal sizes: In order to have enough data to fit, 12 earthquakes (Fig. 1b) fitting a given criterion were chosen from 40 catalogued earthquakes with magnitude greater than 5.0 occurring in Australia after 1980. The empirical criterion used here is that there are more than 30 events with magnitudes greater than 2.5 and within $200 \mathrm{~km}$ of the epicenter of a main earthquake recorded in the catalog. The results show that 7 events are preceded by AMR, and yield very good fits to (1) with high curvature (e.g., $C<0.65, m \sim 0.3$, listed in Table 1). Figure 2 shows plots of the typical cumulative Benioff strain and plots of the best-fit values for $C$ and $m$ versus radius.

Two earthquakes (no. 7 and 10 in Table 1) occurred in similar locations as events of similar magnitudes (no. 5 and 8) within very short-time intervals (around 4 years). Hence the sequences preceding these events were too short to be considered independent from the previous large event (Fig. 2c). There were three other cases (no. 3,4 and 12) in which the best-fit curvature parameter $C$ and exponent $m$ indicated no AMR. In these cases, re-examination indicated that the sequences had relatively little data (no more than 15 events prior to the main event), so it is possible that data paucity was responsible for the poor fits (e.g., $m>0.8, C>0.7$ ). 

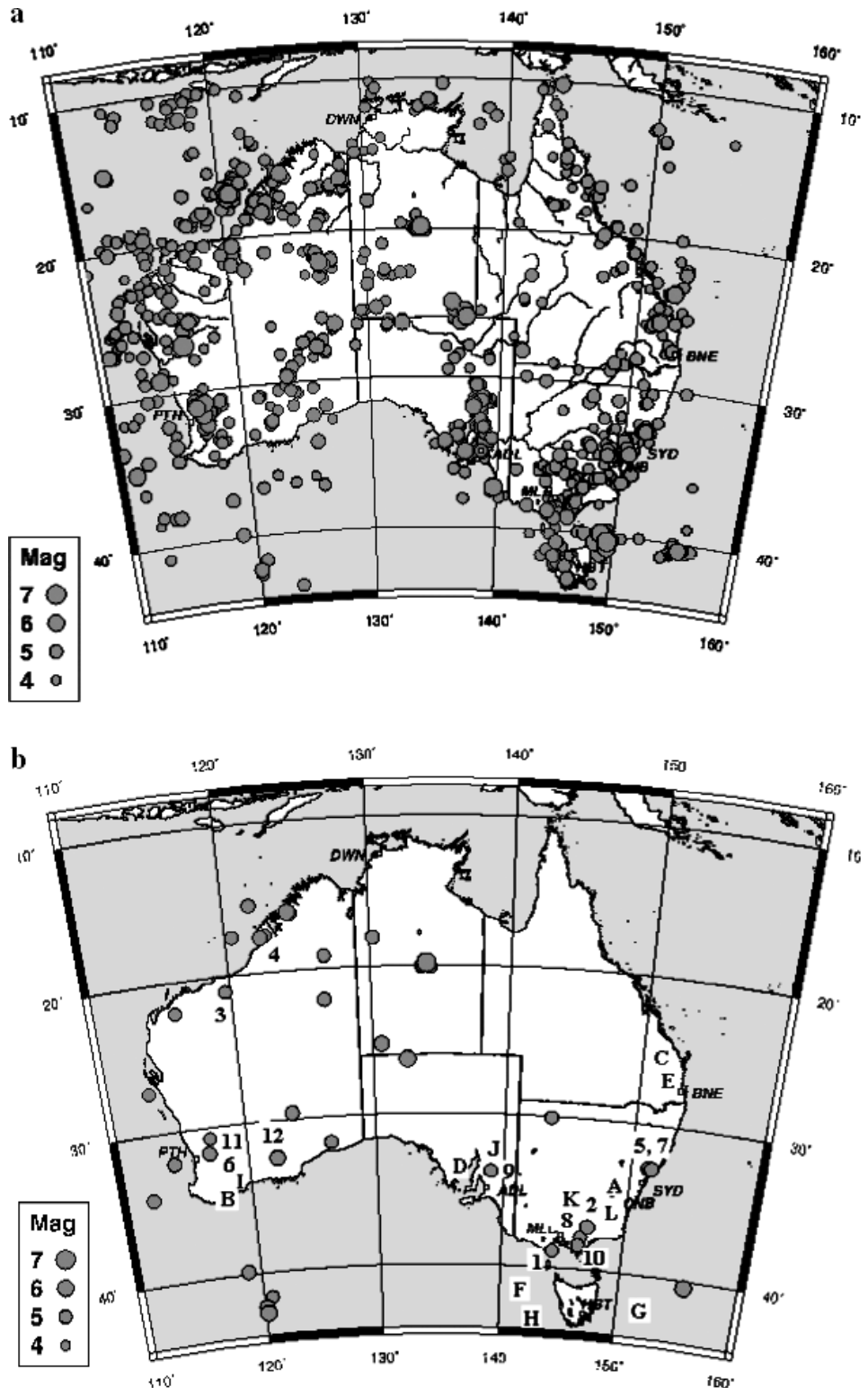

Figure 1

Australian $M>4.0$ earthquakes since 1960 (a), and 12 events with good data, labeled with numbers (b). The catalog originates from Geoscience Australia.

Optimal radius versus magnitude: Since our data cover a very limited magnitude range $(5.0 \sim 5.5)$, we plotted our results together with the results of other researchers in Figure 3. The plots support the idea that the critical region size scales with the 
Table 1

Twelve earthquakes chosen from 40 catalogued earthquakes with magnitudes greater than 5.0 occurring in Australia after 1980 and the fitting parameters

\begin{tabular}{ccccccccc}
\hline No. & Time & Long. & Lat. & Mag. & AMR or not $?$ & $\mathrm{M}$ & $\mathrm{C}$ & $\mathrm{R}_{\mathrm{c}}(\mathrm{km})$ \\
\hline 1 & $1981 / 6 / 16$ & 144.20 & -38.90 & 5.1 & Yes & 0.25 & 0.45 & 125 \\
2 & $1982 / 11 / 21$ & 146.96 & -37.20 & 5.4 & Yes & 0.42 & 0.60 & 150 \\
3 & $1988 / 1 / 28$ & 119.60 & -21.05 & 5.0 & No, few data & & & \\
4 & $1989 / 10 / 13$ & 122.43 & -17.64 & 5.4 & No, few data & & & \\
5 & $1989 / 12 / 27$ & 151.61 & -32.95 & 5.5 & Yes & 0.25 & 0.55 & 175 \\
6 & $1990 / 1 / 17$ & 116.99 & -31.72 & 5.5 & Yes & 0.25 & 0.62 & 125 \\
7 & $1994 / 8 / 6$ & 151.29 & -32.92 & 5.3 & No, too close to no. 5 & & & \\
8 & $1996 / 9 / 25$ & 146.47 & -37.88 & 5.0 & Yes & 0.36 & 0.55 & 200 \\
9 & $1997 / 3 / 5$ & 138.97 & -33.82 & 5.0 & Yes & 0.62 & 0.65 & 100 \\
10 & $2000 / 8 / 29$ & 146.29 & -38.41 & 5.0 & No, too close to no. 8 & & & \\
11 & $2001 / 9 / 28$ & 117.00 & -30.50 & 5.1 & Yes & 0.47 & 0.60 & 100 \\
12 & $2001 / 10 / 20$ & 120.60 & -33.60 & 5.2 & No, few data & & & \\
\hline
\end{tabular}

magnitude of the earthquake, and the best-fitting least-squares line for the total data set is $\log R_{c}=-0.37+0.38 M$. It is noticeable that our critical sizes are systematically larger than would be predicted by the best-fitted line for the similar magnitudes, indicating a longer fault interaction distance in the Australian continent. A possible explanation is that the fault system is more stretched in intra-plate regions than in inter-plate regions.

Fitting time $\Delta t=t_{f}--t_{0}$ : In Figure 4 we plot our results of fitting time versus magnitude together with the results of other studies. No systematic scaling is observed. However, except 6 points with the smallest $\Delta t$ and one point with the very largest $\Delta t$, a linear trend can still roughly be seen for most other points. Fitting time may depend on several complicated factors, such as loading rate of the tectonic stress (PApazachos and Papazachos, 2000), heterogeneity of the focus media and even quality of data. Also, it should be constrained by the recurrence time of the similar magnitude in the same region. For instance, in Western Australia, the time period for the $M 5$ events is about 10 years, which should be a good choice for the upper limit of AMR fitting period.

AMR Fitting in the Region where there are no Earthquakes with Magnitude above 5.0

In order to investigate how the cumulative Benioff release looks in areas without large events, we randomly chose 12 points with enough data for the analysis but no large events during a curtain period, and determined the fit to Eq. (1). The results show that 10 out of 12 regions have no acceleration of Benioff release, and $C$ and $m$ values are larger than 0.6 , mostly exceeding 0.7 . The remaining two cases have good fits, which means false alarms would have been triggered using the AMR method. 

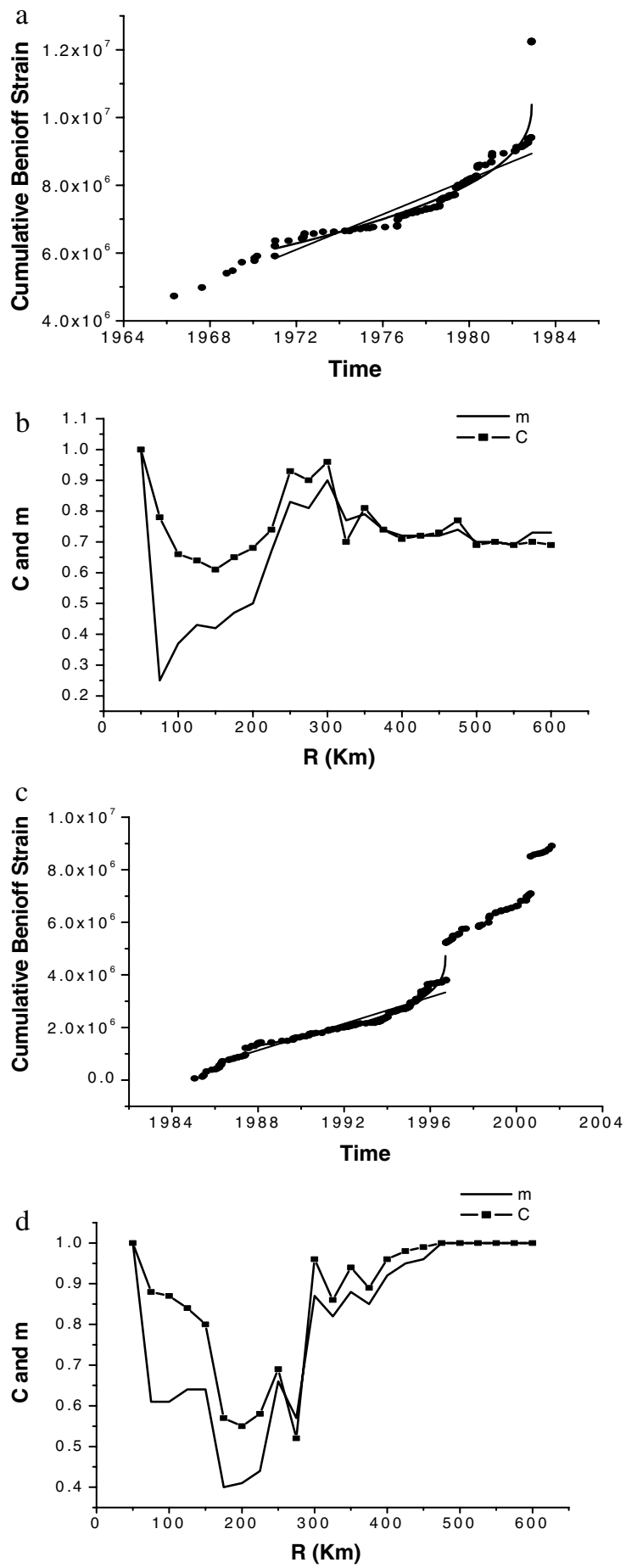


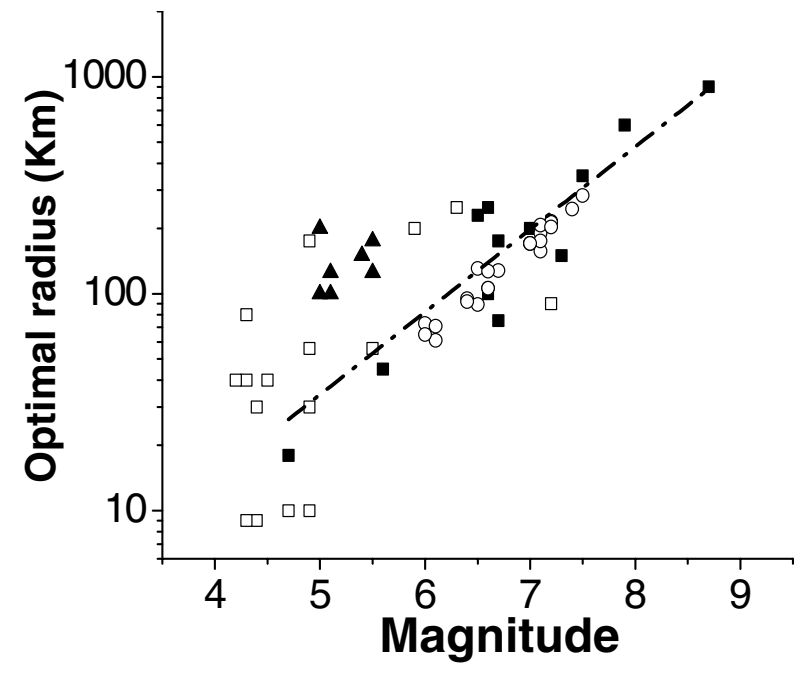

Figure 3

Relation between magnitude and optimal radius. Solid squares are results from Bowman et al., (1998), open circles are from Papazachos and PAPAzachos (2000), open squares are from Brehm and Braile (1998), and solid triangles are our data. The total data set is best-fitted to $\log R_{c}=-0.37+0.38 M$ shown by the dotted line.

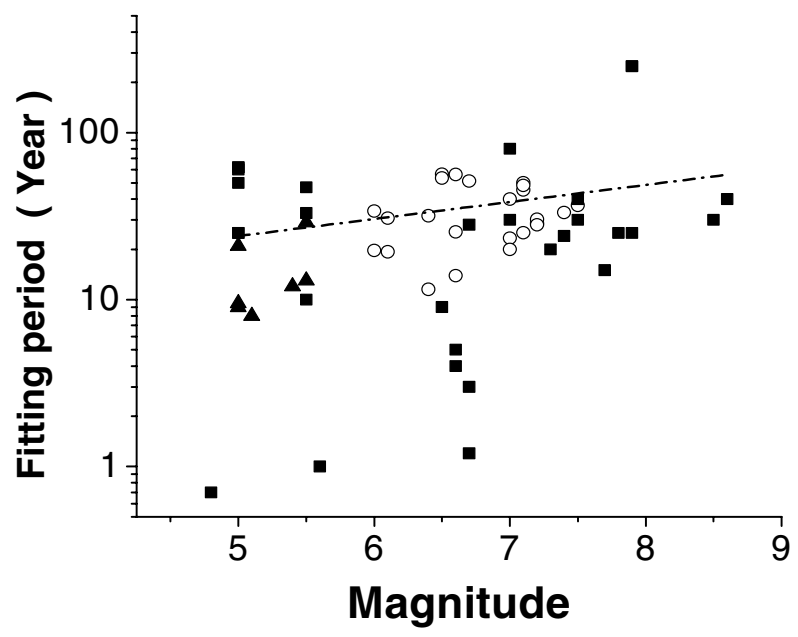

Figure 4

Plots of the fitting time period versus magnitude. Open squares are data from BUFE and VARNES (1993), BUFE et al., (1994) and Robinson (2000), open circles are from PAPAZACHOS and PAPAZAChOS (2000), and solid triangles are our data. The dotted line, $\log \Delta t=0.869+0.102 \mathrm{M}$, shows the best-fitted line excluding 7 points.

Figure 2

Typical cumulative Benioff strain and AMR fit ( $2 \mathrm{a}$ and 2c), and best fit $C$ (the goodness-of-fit parameter) and $m$ (curvature exponent) versus radius ( $2 \mathrm{~b}$ and $2 \mathrm{~d}$ ) for 2 events in Table $1.2 \mathrm{a}$ and $2 \mathrm{~b}$ are for the event no. 2 and $2 \mathrm{c}$ and $2 \mathrm{~d}$ for the event no. 8 . 


\section{Spatio-temporal Scanning of AMR Parameters}

To ascertain how many AMR anomalies large earthquakes follow, we performed a spatio-temporal scan of AMR in Australia from 1980. The spatial range covers longitudes from 110 to 155 and latitudes from -45 to -11 . A spatial interval of 0.2 degrees was used in the scanning, a time interval of 3 months was used for $t_{f}$, and a radius of $\mathrm{R}=125 \mathrm{~km}$ was used when selecting data from the earthquake catalog for analysis at each point. Since single large earthquakes can cause an apparent AMR anomaly, we neglect such anomalies in the following summary. In total, 24 AMR regions are detected and earthquakes above magnitude 4.5 follow in 20 of these regions. The remaining four cases are false alarms. It is possible that the false alarms are partly due to the uniform parameters for all points in the scanning (such as the fixed radius of $125 \mathrm{~km}$ ) and the circular regions used to select the events. Further work would be required in order to test this possibility.

\section{Relation between AMR and LURR}

The Load-Unload Response Ratio (LURR) method is an intermediate-term earthquake prediction approach that has shown considerable promise. The basic assumption of LURR is that when a system is stable, it is not sensitive to small external perturbations, whereas when the system is in an unstable state, the response to loading and unloading becomes quite different. LURR was proposed to measure this difference, and was used as an indicator of a system approaching critical state. In practice, this method typically involves calculating the ratio of Benioff strain release during periods of loading and unloading as determined by calculating earth tide induced perturbations in the Coulomb Failure Criterion on optimally oriented faults. In retrospective studies, high LURR values have been observed months to years prior to most events and intermediate-term earthquake predictions have been made (YIN et al., 1995, 2000).

Since both AMR and LURR can be used as indicators that the crust within a region of study is approaching a critical state, questions arise: Do these observations correlate with one another? Do AMR and LURR predict a similar time, magnitude and critical region size prior to a large earthquake?

Sizes of critical region: For each of the 7 earthquakes we calculated AMR and LURR as well as the optimal radius (Fig. 5). We found that the optimal radius for LURR (corresponding to maximum LURR value) is similar to the optimal radius for AMR (corresponding to minimum $C$ and $m$ ).

Predicted critical time: By comparing LURR versus time plots with plots of $C$ and $m$ versus time (Fig. 6), we found that earthquakes occur 1.2 to 3.2 years (average 1.9) after the LURR value begins to rise, and 0.3-2 years (average 1) after the LURR value reaches its maximum. In contrast, for $m$ and $C$ values, earthquakes mostly occur 1.7-12 years after $C$ and $m$ values begin to decrease (the average is 3.5 years excluding the largest value of 12), and 1 to 9 years (the average is 1.3 years excluding 


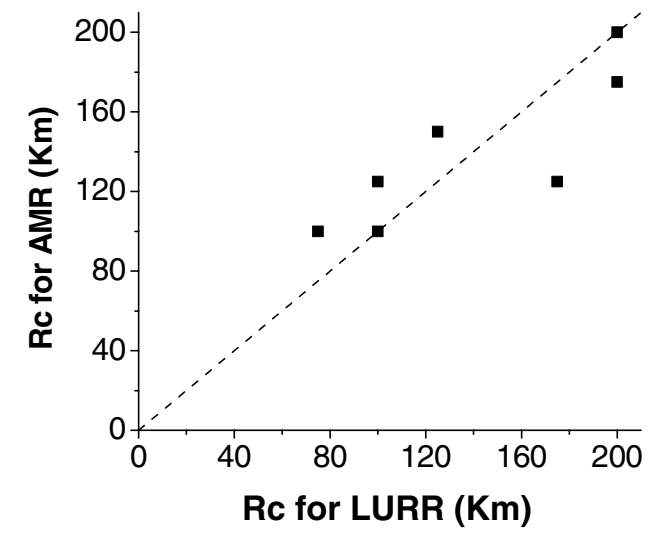

Figure 5

The optimal radii for LURR versus the optimal radii for AMR. The dotted line shows $R_{c L U R R}=R_{c A M R}$.
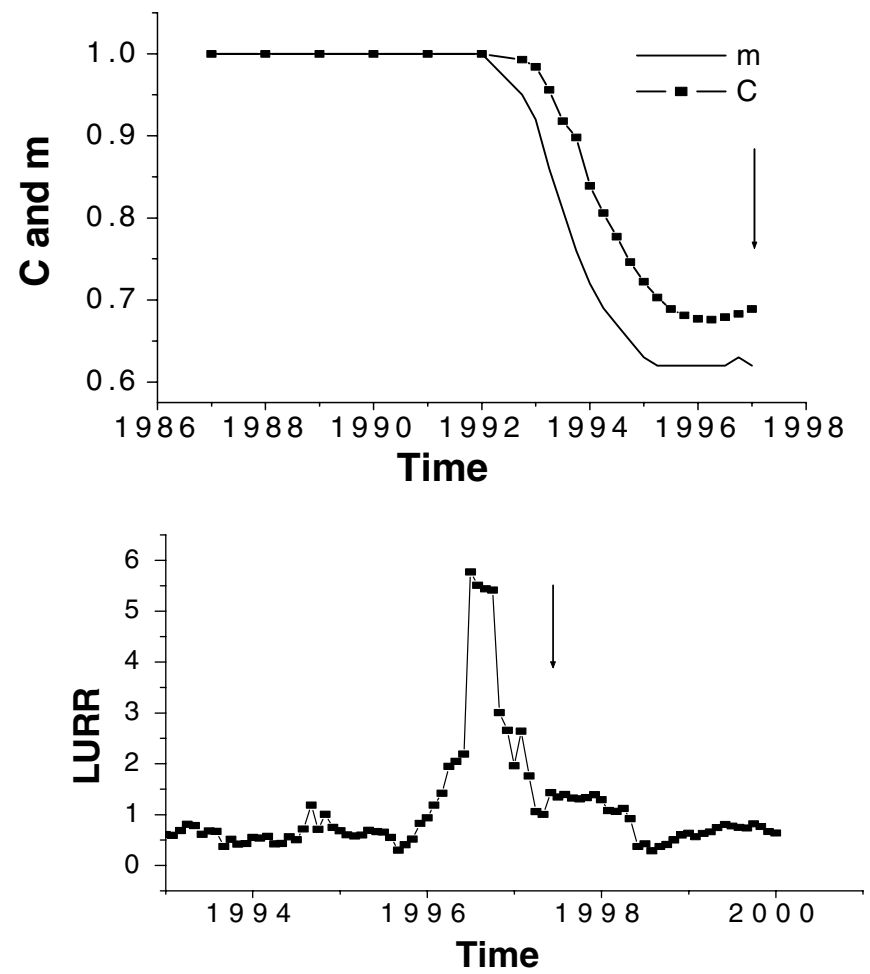

Figure 6

Plot of $C$ and $m$ (top) and LURR values versus time (bottom) before the earthquake on 5 May, 1997 (no. 9 in Table 1). The arrow indicates when the above magnitude 5 earthquake occurred. 
the largest value of 9) after $C$ and $m$ values reach a minimum. Hence AMR predictions for $t_{f}$ appear to occur a little earlier than LURR peaks, or equivalently, the LURR method appears to provide a shorter-term prediction than the AMR method.

\section{Discussion and Conclusions}

The results of this study provide support for use of the AMR model for earthquake forecasting and for the Critical Point Hypothesis. Our results show that seven of the large events with sufficient data (defined here as more than 15 events within the optimal radius) are preceded by accelerating Benioff strain release. The results also suggest that if two events of similar magnitude occur in the same region within a short-time interval, the AMR model will fail to predict the second one, possibly due to interference with the first event.

The optimal region size for AMR scales with the magnitude, in agreement with previous results, however, the critical sizes in Australia are slightly larger than those in California. This may be related to the difference between intra-plate fault systems and inter-plate fault systems. No apparent relation between the fitting time period and the magnitude is found, suggesting that the fitting time period may depend strongly on the particular region under consideration. It should be constrained by the recurrence time of similar magnitude events in that area.

In regions of similar seismicity without large earthquakes but with enough data for the analysis, most cases (10 out of 12) do not exhibit AMR and an optimal region size cannot be determined. Two false alarms were found where AMR was not followed by a large event. In a spatio-temporal scanning study earthquakes follow $83 \%$ of AMR anomalies and $17 \%$ were found to be false alarms which may be due in part to uniform parameters and circular regions we used.

LURR and AMR predict a similar critical region size, but the critical time predicted by AMR is observed to be slightly earlier than the time of LURR peaks. The mechanisms for AMR and LURR require better understanding to comprehend the significance of this observation.

Although AMR has some predictive capability, further research is required in order to apply AMR to the practice of earthquake prediction. In the present study, most of the $M>5$ events cannot be tested using the time-to-failure formula due to insufficient data, thus raising several questions. The first is: Do these earthquakes which can not be fit well really have no AMR beforehand or is it a symptom of incomplete catalogs due to too few seismograph stations nearby? If it is a catalog problem, then improved monitoring may decrease the failure rate of AMR. However, if there are really no AMRs prior to those events, different physical mechanisms need to be proposed. At present, it is difficult to tell the difference between $M>5$ events which cannot be tested and those which can, since we have 
inferior records around those events and sparse knowledge regarding the tectonic settings. Possible clues may be gained in the regions with dense networks and good records.

Another interesting question is: In what circumstances does AMR appear prior to the large events? According to JAUMÉ and SyKes (1999), three possible necessary conditions for AMR are: a certain degree of heterogeneity in the fault system, the density of faults or asperities, and the presence of a sufficiently large earthquake. Another potential factor may be the loading rate of tectonic stress. It is suggested by VIDALE et al. (1998) that preseismic stress rates in fault zones are much higher than long-term tectonic stress rates. Does this high stress rate within the fault zone contribute to AMR? In other words, is AMR mainly caused by relatively high stress rates or stress evolution under constant or low loading rates? What kind of parameters control $C$ and $m$ in Equation (1), and what controls the optimal size $R_{c}$ ?

The relationship between LURR and AMR deserves further study. For example, what percentage of large earthquakes is preceded by both phenomena? How many earthquakes are preceded by only one or none of these phenomena? While they are both observed just prior to a large earthquake, one could ask, is high LURR generally caused by increased seismicity in loading cycles, or by decreased seismicity in unloading cycles, or increased seismicity in both cycles, but at a consideably faster rate in loading cycles? What is the mechanism responsible for this? Is there a common physical basis behind AMR and LURR as suggested by the common critical scaling region (YIN et al., 2002)? These questions could be studied further using good quality earthquake data or using numerical simulations.

\section{Acknowledgements}

This study is supported by the Australia-China special Fund for Scientific and Technological Cooperation, Chinese NSF Fund for International Exchange and Cooperation and Chinese NSF (Grant num. 40004002). We are also grateful to Dr. Cvetan Sinadinovski of Geoscience Australia (formerly known as Australian Geological Survey Organization - AGSO) for providing the Australia catalog. The GMT Software (WESSEL and SMITH, 1995) was used in this study. The authors are grateful for Dr. David Bowman 's valuable suggestions enhancing the manuscript.

\section{REFERENCES}

BAK, P. and TAng, C. (1989), Earthquakes as a Self-organized Critical Phenomenon, J. Geophys. Res. 94 , 15,635-15,637.

Bowman, D. D., Oulllon, G., Sammis, C. G., Sornette, A., and Sornette, D. (1998), An Observational Test of the Critical Earthquake Concept, J. Geophys. Res. 103, 24,359-24,372. 
Bowman, D. D. and KIng, G. C. P. (2001), Accelerating Seismicity and Stress Accumulation before Large Earthquakes, Geophys. Res. Lett. 28, 4039-4042.

Breham, D. and Braile, L. W. (1998), Intermediate-term Earthquake Prediction Using Precursory Events in the New Madrid Seismic Zone, Bull. Seismol. Soc. Am. 88, 564-580.

Breham, D. and Braile, L. W. (1999), Intermediate-term Earthquake Prediction Using the Modified Timeto-failure Method in Southern California, Bull. Seismol. Soc. Am. 89, 275-293.

Bufe, C. G. and Varnes, D. J. (1993), Predictive Modeling of the Seismic Cycle of the Greater San Francisco Bay region, J. Geophys. Res. 98, 9871-9883.

Bufe, C. G. Nishenko, S. P., and Varnes, D. J. (1994), Seismicity Trends and Potential for Large Earthquakes in the Alasks-Aleutian Region, Pure Appl. Geophys. 142, 83-99.

Geller, R. J., Jackson, D. D., Kagan, Y. Y., and Mulargia, F. (1997), Earthquakes Cannot be Predicted, Science 275, 1616-1617.

Huang, Y., Saleur, H., Sammis, C., and Sornette, D. (1998), Precursors, Aftershocks, Criticality and Self-organized Criticality, Europhys. Lett. 41,43-48.

Ito, K. and Matruzaki, M. (1990), Earthquakes as a Self-organized Critical Phenomenon, J. Geophys. Res. 95, 6853-6860.

Jaumé, S. C. and Sykes, L. R. (1999), Evolving Towards a Critical Point: a Review of Accelerating Seismic Moment/Energy Release Prior to Large and Great Earthquakes, Pure Appl. Geophys. 155, 279-306.

Karakaisis, G. F., Papazachos, B. C., Papazachos, C. B., and Savvaidis, A. S. (2002), Accelerating Seismic Crustal Deformation in the North Aegean Trough, Greece, Geophys. J. Int. 148, 193-200.

Keilis-Borok, V. I., Knopoff, L., Rotwain, I. M., and Allen C. R. (1988), Intermediate-term Prediction of Occurrence Times of Strong Earthquakes, Nature 335, 690-694.

Knopoff, L., Levshina, T., Keillis-Borok V. I., and Mattoni, C. (1996), Increased Long-range Intemediate-magnitude Earthquake Activity prior to Strong Earthquakes in California, J. Geophys. Res. 101, 5779-5796.

Ouillon, G. and Sornette, D. (2000), The Concept of 'Critical Earthquake' Applied to Mine Rockbursts with Time-to-failure Analysis, Geophys. J. Int. 143, 454-468.

Papazachos, B. and Papazachos, C. (2000), Accelerating Preshock Deformation of Broad Regions in the Aegean Area, Pure Appl. Geophys. 157, 1663-1681.

Robinson, R. (2000), A Test of the Precursory Accelerating Moment Release Model on Some Recent New Zealand Earthquakes, Geophys. J. Int. 140, 568-576.

Saleur, H., Sammis, C. G., and Sornette, D. (1996), Discrete Scale Invariance, Complex Fractal Dimensions, and Log-periodic Fluctuations in Seismicity, J. Geophys. Res. 101, 17,66-17,677.

Sammis, C. G. and Smith, S. W. (1999), Seismic Cycles and the Evolution of Stress Correlation in Cellular Automation Models of Finite Fault Networks, Pure Appl. Geophys. 155, 307-334.

Sornette, A. and Sornette, D. (1989), Self-organized Criticality and Earthquakes, Europhys. Lett. 9, 197.

Sornette, D. and Sammis, C. G. (1995), Complex Critical Exponents from Renormalization Group Theory of Earthquake Prediction, J. Phys. I. France 5, 607-619.

Sykes, L. R. and Jaumé, S. (1990), Seismic Activity on Neighboring Faults as a Long-term Precursor Term Precursor to Large Earthquakes in the San Francisco Bay Area, Nature 348, 595-599.

VArnes, D.J. (1989), Predicting Earthquakes by Analyzing Accelerating Precursory Seismic Activity, Pure Appl. Geophys. 130, 661-686.

Vidale, J. E., Agnew, D. C., Johnston, M. J. S., and Oppenheimer, D. H. (1998), Absence of Earthquake Correlation with Earth Tides: An Indication of High Preseismic Stress Rate, J. Geophys. Res. 103, 24,567-24,572.

Wessel, P. and Smith, W. H. F. (1995), New Version of the Generic Mapping Tools Released, EOS Trans. Am. Geophys. Union 76, 329.

Yin, X. C., Chen, X. Z., Song, Z. P., and Yin, C., (1995), A New Approach to Earthquake Prediction-the Load-Unload Response Ratio (LURR) Theory, Pure Appl. Geophys. 145, (3/4), 701-715.

Yin, X. C., Wang, Y. C., Peng, K. Y., Bai, Y. L., Wang, H. T., and Yin, X. F. (2000), Development of a New Approach to Earthquake Prediction: Load-Unload Response Ratio (LURR) theory, Pure Appl. Geophys. 157, 2365-2383. 
Yin, X. C., Mora, P., Peng, K. Y., Wang, Y. C., and Weatherley, D. (2002), Load-Unload Response Ratio and Accelerating Moment/Energy Release Critical Region Scaling and Earthquake Prediction, Pure Appl. Geophys. 159, 2511-2523.

(Received September 27, 2002, revised March 20, 2003, accepted March 30, 2003)

To access this journal online:

(20) http://www.birkhauser.ch 